\title{
An overview of the rotational behavior of metal-poor stars
}

\author{
C. Cortés, ${ }^{1}$ J. R. P. Silva, ${ }^{2}$ A. Recio-Blanco,${ }^{3}$ M. Catelan, ${ }^{4}$ \\ J. D. Do Nascimento Jr. ${ }^{1}$ and J. R. De Medeiros ${ }^{1}$ \\ ${ }^{1}$ Universidade Federal do Rio Grande do Norte (UFRN), Brazil \\ ${ }^{2}$ Universidade Estadual do Rio Grande do Norte (UERN), Brazil \\ ${ }^{3}$ Observatoire de la Côte d'Azur, France \\ ${ }^{4}$ Pontifícia Universidad Católica, Chile
}

\begin{abstract}
We describe the behavior of the rotational velocity in metal-poor stars $([\mathrm{Fe} / \mathrm{H}]$ $\sim-0.5$ dex) at different evolutionary stages, based on $v \sin i$ values from the literature. Our sample is composed of stars in the field and in some Galactic globular clusters, including stars on the main sequence (MS), red-giant branch (RGB), and horizontal branch (HB). The metalpoor stars are mainly slow rotators, and their $v \sin i$ distribution along the Hertzsprung-Russell diagram is quite homogeneous. Nevertheless, a few moderate to high values of $v \sin i$ are found for stars located on the MS and the HB. We show that the overall distribution of $v \sin i$ values is basically independent of metallicity for the stars in our sample. In particular, the fast-rotating MS stars in our sample exhibit similar rotation rates as their metal-rich counterparts, suggesting that some may actually be fairly young, in spite of their low metallicity, or else that at least some would be better classified as blue straggler stars. We do not find significant evidence of evolution in $v \sin i$ values as a function of position on the RGB. In particular, we do not confirm previous suggestions that stars close to the RGB tip rotate faster than their less-evolved counterparts. While the presence of fast rotators among moderately cool blue-HB stars has been suggested as due to angular-momentum transport from a stellar core that has retained significant angular momentum during its prior evolution, we find that any such transport mechanisms must likely operate very fast as the star arrives on the zero-age HB (ZAHB), since we do not find a link between evolution off the ZAHB and $v \sin i$ values.
\end{abstract}

Keywords. stars: evolution, stars: fundamental parameters, stars: Population II, stars: rotation, stars: statistics

The full poster (in pdf format) is available at http: //www . astro.iag.usp.br/ iaus266/Posters/pCortes.pdf. 